\title{
Analysis of Characteristics of the Forecast Jump in the NCEP Ensemble Forecast Products
}

\author{
Xiakun Zhang1, Liping Zhang ${ }^{2 *}$, Jiao Fuㄹ, Longxi Zhang4 \\ ${ }^{1}$ National Meteorological Center of CMA, Beijing, China \\ ${ }^{2}$ Shanxi Meteorological Administration Training College, Taiyuan, China \\ ${ }^{3}$ Meteorological Bureau of Xingtai City, Xingtai, China \\ ${ }^{4}$ College of Science, Minzu University of China, Beijing, China \\ Email: ${ }^{\star}$ zhangliping365@163.com
}

How to cite this paper: Zhang, X.K., Zhang, L.P., Fu, J. and Zhang, L.X. (2017) Analysis of Characteristics of the Forecast Jump in the NCEP Ensemble Forecast Products. Atmospheric and Climate Sciences, 7, 151159.

http://dx.doi.org/10.4236/acs.2017.71011

Received: October 14, 2016

Accepted: January 22, 2017

Published: January 25, 2017

Copyright $\odot 2017$ by authors and Scientific Research Publishing Inc. This work is licensed under the Creative Commons Attribution International License (CC BY 4.0).

http://creativecommons.org/licenses/by/4.0/

\section{Open Access}

\begin{abstract}
The limit of numerical prediction and ensemble prediction can be further understood by the study of the forecast jump. By using the ensemble average forecast and control forecast product output data for the United States National Environmental Prediction Center (NCEP) global ensemble forecast system (GEFS), and the concept of Jumpiness index from Zsoter et al., we analyzed the statistical characteristics of forecast jump. Results show that, on average, in the NCEP ensemble forecast product, the time average prediction jump index increases with the increase of the forecast aging, and the actual forecast experience can reflect this phenomenon. The consistency of ensemble average forecast is better than the corresponding control forecast. Also, in summer, the frequency of "forecast jump" phenomenon is fluctuating by $17.5 \%$.
\end{abstract}

\section{Keywords}

Forecast Jump, Flip-Flop-Flip, NCEP Ensemble Forecast Products, Analysis

\section{Introduction}

With the rapid development of numerical forecast and the ensemble forecast, many problems to be solved also appear, and these questions are included in the "forecast jump" [1] [2]. "Forecast jump" is also known as "forecast inconsistency" or "forecasts discontinuity", which is a big difference between any two or more times of the multiple continuous forecasting with different forecasting start times and the same forecasting moment time [2] [3] [4].

Because the numerical prediction technology is not perfect yet, it is hard to avoid forecast jump, which not only seriously affects the forecasters' judgment on the forecast results, but also undermines user's confidence in weather forecasts. Its harm is very ob- 
vious [5] [6] [7] [8]. Especially for the strong convection, heavy rain and other severe weather events, if there is a forecast jump, it will seriously affect the forecast and the user's decision-making, leading to the loss of the severe weather. Therefore, the forecast of jumping in-depth study is very necessary, and the limit of numerical prediction and ensemble prediction can be further understood by the study of the jump. Then we can improve the numerical forecast and ensemble forecast system, promote the forecasting accuracy rate unceasing enhancement, and enhance the confidence of users for numerical forecast products. Finally, we will provide a more scientific basis for reliable decision-making in user's dealing with severe weather events.

At present, the studies of forecast jump just begin, and most of the research work is focused on the analysis of the NCEP and ECMWF and UKMO ensemble forecast product performance in North America or Europe [8] [9]. Recently, the European center through the establishment of "jump forecast index" method to forecast jumping question has carried on the quantitative research, and tentatively explored the causes of forecast jump on the basis of quantitative study [10] [11] [12]. Study forecast leap in Asia, however, has not been deeply involved yet.

In view of the above questions, we will use the NCEP ensemble mean forecast and control forecast product data to carry on the statistical analysis and the contrast research on its forecast jump in the Asian region in this paper. Through the study of this chapter, it is helpful to recognize the forecast jump features of NCEP ensemble forecast, and the forecaster's ability of releasing with NCEP ensemble forecast products was improved, in order to provide more high quality weather forecast service to the users.

\section{Data and Methods}

The data used in this paper is the ensemble average forecast and control forecast product output data for the United States National Environmental Prediction Center (NCEP) global ensemble forecast system (GEFS). The forecast factors are selected as $500 \mathrm{hPa}$ height field, forecast the starting time is on March 1, 2011 to February 28, 2013, a total of 731 days. Forecast start time is 00 and 12 times per day (the same below), the forecast time is 6 hours. Forecast area is $15^{\circ} \mathrm{N}-18^{\circ} \mathrm{N}$ and $40^{\circ} \mathrm{E}-160^{\circ} \mathrm{E}$, the horizontal spatial resolution is $1^{\circ} \times 1^{\circ}$ latitude and longitude. This area was selected based on GEFS' application of Northwest China.

At present, it is usually used to make objective and quantitative analysis of the forecast jump by the forecast jump index. In this paper, one of forecast jump index, Jumpiness index can be used to analyze and compare the forecast jump. Zsoter et al. first proposed the concept of Jumpiness index [2], and give the definition of Jumpiness index type:

$$
\mathrm{INC}_{\Sigma}[f(d, t), \delta]=\frac{\operatorname{diff}_{\Sigma}[f(d, t), f(d+\delta, t-\delta)]}{0.5\left\{\operatorname{std}_{\Sigma}[f(d, t), \delta]+\operatorname{std}_{\Sigma}[f(d+\delta, t-\delta)]\right\}} .
$$

In the formula, $f$ denotes forecast fields, $d$ and $d+\delta$ denote starting time forecasts, $t$ and $t-\delta$ denote prediction time, $\delta$ denote repay twice the time interval, in this study, for 12 hours. In this way, the forecast time of these two forecasts are $d+t$. Therefore this article says $f(d, t)$ and $f(d+\delta, t-\delta)$ prediction results of adjacent prediction 
start times. Subscript $\Sigma$ representation of the selected area forecast regional average operation, in particular, in Equation (1) the right of the molecule $\operatorname{diff}_{\Sigma}[f(d, t), f(d+\delta, t-\delta)]$ show the difference between the two prediction results of $t-\delta$ and $T$, respectively; in Equation (1) the right side of the denominator expressed respectively forecast time $t$ and $t-\delta$ the standard deviation of two forecast arithmetic average operations, the introduction of the standard deviation of the jump to forecast index standardized treatment. Standardization can make different forecast system of forecast jump index to compare directly.

The time averaged prediction jump index [2] is defined as

$$
\left\langle\mathrm{INC}_{\Sigma}[f(t), \delta]\right\rangle_{N}=\sqrt{\frac{1}{N} \sum_{d=1}^{N}\left\{\mathrm{INC}_{\Sigma}[f(d, t), \delta]\right\}^{2}} .
$$

In Equation (2), $\left\langle\mathrm{INC}_{\Sigma}[f(t), \delta]\right\rangle_{N}$ is jump forecast index of root mean square with $N$ times. To forecast jump phenomena, the emergence of "flip" phenomenon is defined as to half of the jump time average forecast index as the critical value, the absolute value of prediction index jumping phenomenon more than the critical value. On the basis of the definition of "flip" phenomenon, it continues to define "flip-flop" and "flip-flop-flip" phenomenon. It is defined as "the flip-flop", for a future in the same moment of forecast, forecasting the continuous found many times forecast of different starting moment occurred on the forecast of two adjacent to the starting time of forecast jump phenomena, and two times for the above forecast starting moment adjacent forecast jumping phenomenon, they calculated by jumping index is the opposite of the positive and the negative of the forecasts. "Flip-flop-flip" phenomenon is the adjacent three consecutive forecast has forecast jump, jump and arbitrary two adjacent forecast its forecast index sign instead.

Figure 1 shows schematic diagram of the three forecast jump phenomena. For any forecast time, such as point $\mathrm{A}, \mathrm{B}$ and $\mathrm{C}$ forecast jump index is beyond the critical value,

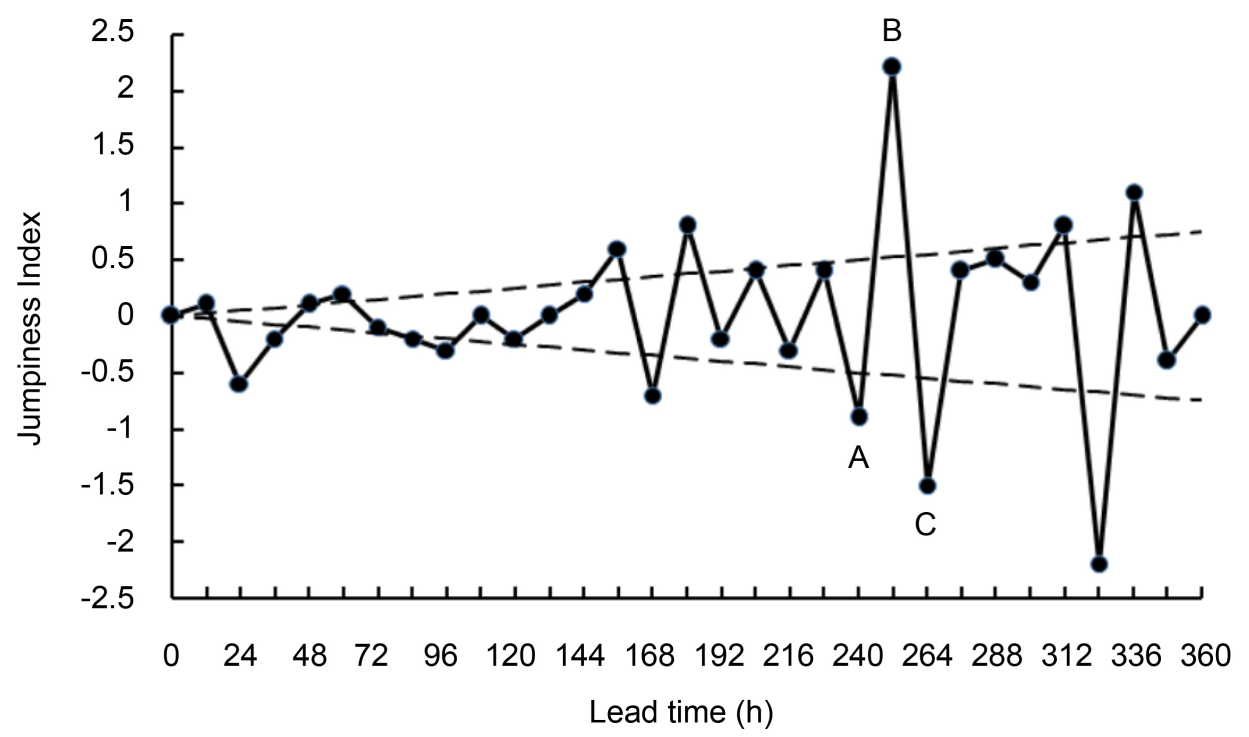

Figure 1. Schematic diagram of three kinds of prediction jump phenomena (point A, B and C indicate a "flip", the dot lines indicate the critical value of the jump exponent). 
so this article will point A, B and C are referred to as a "flip"; for any two adjacent forecast jump index exceeds the critical value of the point, such as point $A$ and $B$ or points $B$ and $C$, to get two changes from point $A$ to point $B$ or from point $B$ to point $C$, the call for a "flip-flop"; for the forecast of three adjacent jump index exceeds the critical value of the point, such as $\mathrm{A}, \mathrm{B}$ and $\mathrm{C}$, for the change from point $\mathrm{A}$ to point $\mathrm{B}$ and then to $\mathrm{C}$, this article said the change as a "flip-flop-flip".

\section{Statistical Characteristics and Analysis of Forecast Jump}

According to this paper, the definition and the calculation formula of the jump index are given, the NCEP ensemble average forecast and the corresponding control forecast are calculated respectively, and the calculation results are shown in Figure 2.

The results of Figure 2 show, in NCEP ensemble forecast product, on average, the predicted jump level increases with the increase of the prediction time, this result is consistent with the forecast practice, and that is longer than the limitation of prediction results to compare the forecast results which are more likely to have on the limitation of short forecast jumps. At the same time, also can be found from Figure 2, for the NCEP ensemble forecast product, the average time of ensemble average forecast is usually smaller than that of the time average of the corresponding control forecast. When the forecast time is relatively long time (more than $144 \mathrm{~h}$ ), this phenomenon becomes more obvious; when the forecast period is not less than $240 \mathrm{~h}$, the average time of the ensemble average forecast is only the time average of the control forecast which corresponds to that of the jump index $25 \%$ - 50\%; However, when the forecast time is relatively short, average time jumping index forecast of ensemble average forecast and control forecast is not very big difference. The above phenomenon can preliminary showed that, don't consider a few special cases, forecast jump can be avoided in a long time by ensemble average forecasting. With the extension of forecasting aging, the forecast jump index of ensemble average is relatively slow. To sum up, the NCEP collection average forecast than its corresponding control has better prediction consistency.

According to the relevant definitions given in this paper, the frequency of "flip",

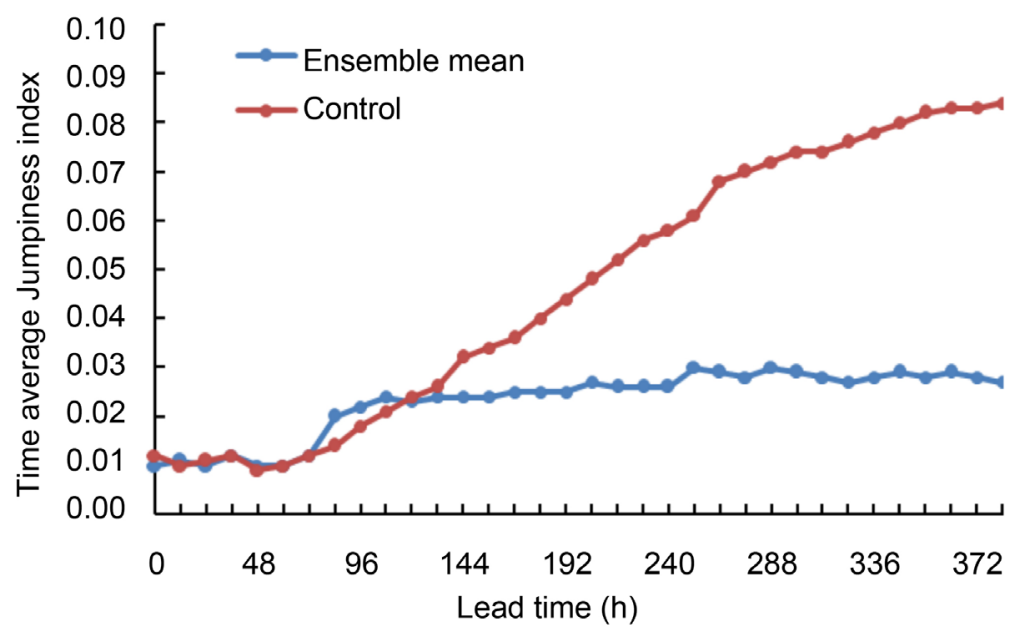

Figure 2. NCEP ensemble mean and control forecast time average forecast jump index. 
"flip-flop" and "flip-flop-flip" phenomena in the ensemble average prediction in the NCEP ensemble forecast products are calculated respectively, the frequency of occurrence of "flip", "flip-flop" and "flip-flop-flip" phenomena occurred in the control and prediction of the NCEP ensemble forecast products, As well as the frequency of "flip", "flip-flop" and "flip-flop-flip" phenomena occurred simultaneously in the above two kinds of prediction, The results are shown in Figures 3-5.

Can be seen from Figure 3, in the range of prediction, the frequency of forecast jump "flip" of NCEP ensemble average forecast and control forecast respectively appears weakly increasing with the extension of the forecast period, prediction jump frequency is in the $50 \%-60 \%$ between fluctuations. Meanwhile, the average forecast of ensemble and the corresponding control forecast at the same time appear the frequency of "flip". But it is worth noting that, in short term forecast (e.g. 48 hours), the occurrence of rapid increase in the frequency of "flip" in the simultaneous occurrence of the ensemble average forecast and control forecast. The above phenomena show that there is no direct correlation between the ensemble average forecast when forecast time is longer and the corresponding control forecast can appear forecast jumping "flip", When the

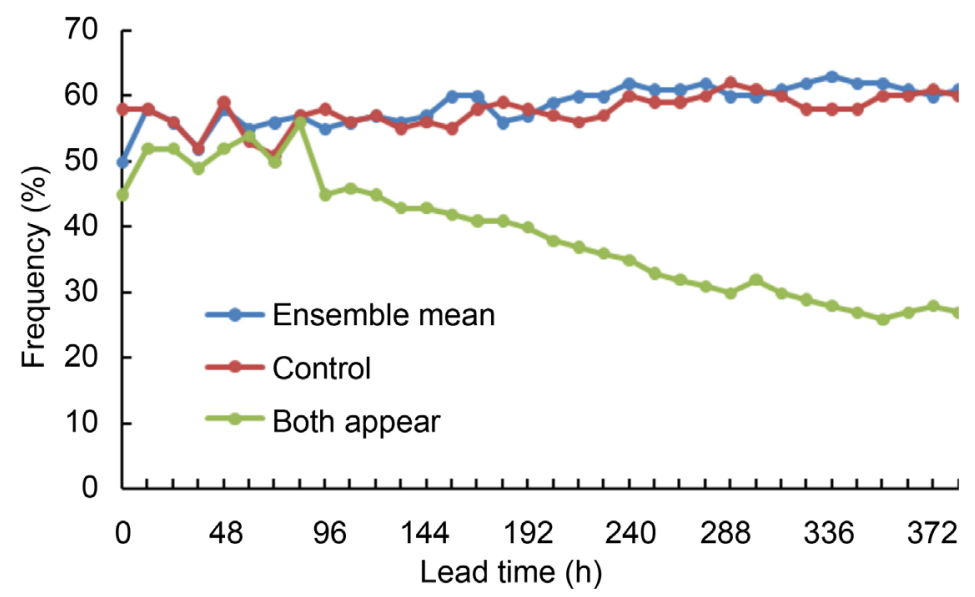

Figure 3. The frequency of "flip" in the NCEP ensemble mean and control forecast.

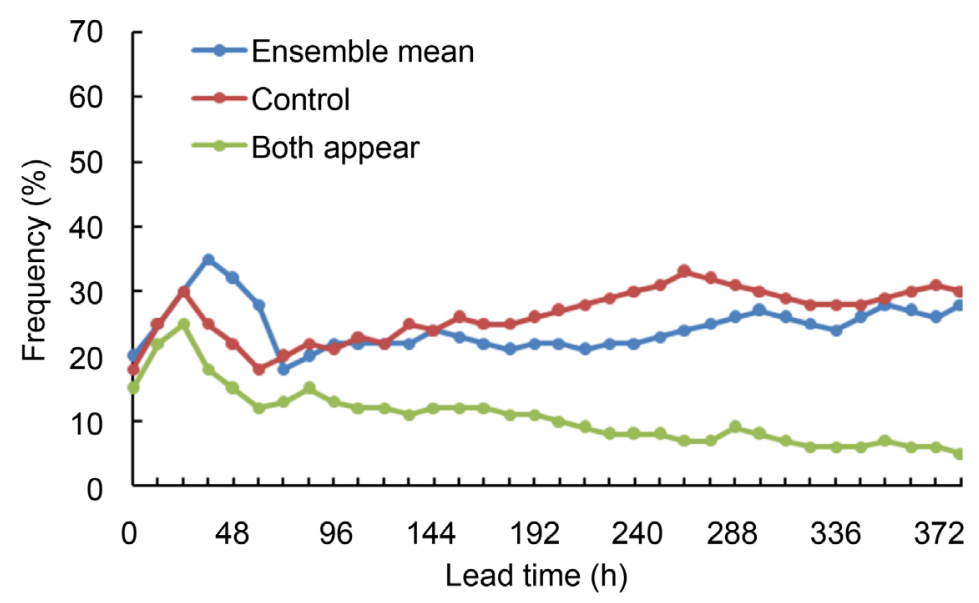

Figure 4. The frequency of "flip-flop" in the NCEP ensemble mean and control forecast. 


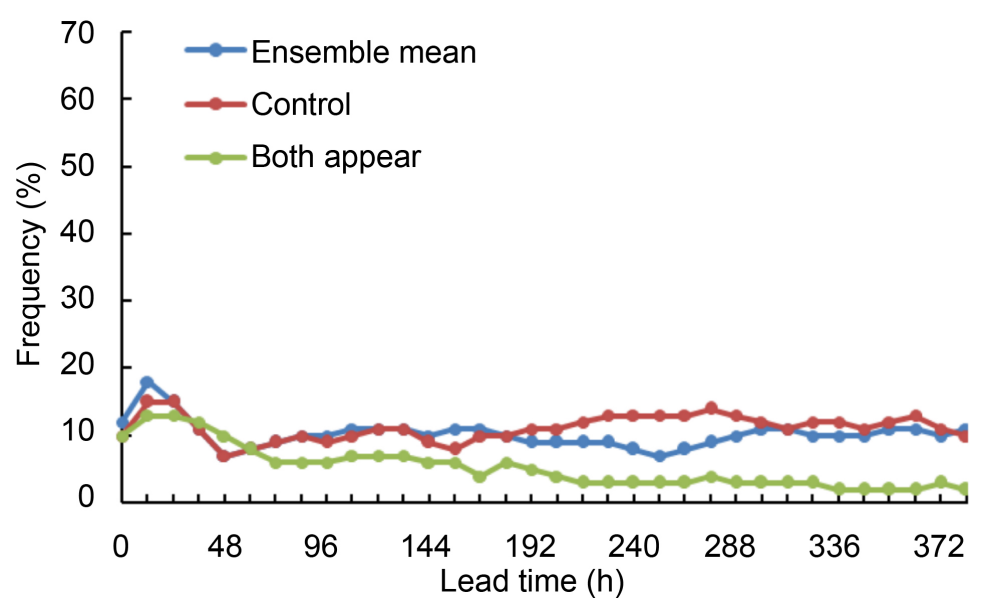

Figure 5. The frequency of "flip-flop-flip" in the NCEP ensemble mean and control forecast.

control prediction, corresponding to ensemble prediction, results are "flip", the ensemble average forecast does not necessarily occur at the same time, "flip", conversely, When the ensemble average forecast appears to predict jump "flip", the control forecast does not appear to predict jump "flip". At the same time, the frequency of "flip" of ensemble average forecast and the corresponding control forecast appearing at the same time is always lower than the frequency of "flip" of ensemble average forecast and the corresponding control forecast appearing alone.

It can be seen from Figure 4, when the forecast time is $0-48 \mathrm{~h}$, the frequency of "flip-flop" appeared by NCEP ensemble average forecast shows rapid growth; when the forecast time is $48-72 \mathrm{~h}$, the frequency of "flip-flop" appears to be reduced quickly; in the range of $72-384 \mathrm{~h}$ forecast, when the forecast time is prolonged, the frequency of "flip-flop" shows a trend of continuous growth in the same time with a slight fluctuation; under the above circumstances, the frequency variation tendency of the control and prediction "flip-flop" is similar to that of the NCEP ensemble average. But it is worth noting that, for the frequency of the appearance of "flip-flop" phenomenon, NCEP ensemble average forecast of the ratio and its corresponding control forecast is slightly lower. When the forecast time is relatively long, the difference between these two frequencies is more obvious. The above analysis indicates that the forecast time is longer, and for the ensemble average forecast and the corresponding control forecast, the forecast jump is different. It can be seen from Figure 4, in the $0-24 \mathrm{~h}$ forecast period, the frequency of the "flip-flop" phenomenon occurs at the same time as the NCEP ensemble average forecast and control forecast, then in the $24-72 \mathrm{~h}$ forecast aging, both at the same time the frequency of the phenomenon of "flip-flop" will be reduced rapidly, at the end of the $72-384 \mathrm{~h}$ forecast period, the frequency is slow down, and the frequency line tends to be gentle; the above frequency variation law similar to the frequency variation law of the "flip-flop" phenomenon in the ensemble average forecast and control forecast, but it is worth noting that the frequency of the "flip-flop" phenomenon occurred simultaneously with the ensemble average forecast and control forecast is much lower than the frequency of the "flip-flop" phenomenon. It can be seen from Figure 5, in the figure can be shown in the $0-384 \mathrm{~h}$ range of the forecast period, 
for the "flip-flop-flip" phenomenon, the changing tendency of their occurrence frequency and the changing tendency of the frequency of the "flip-flop" phenomenon (Figure 4) is similar. However, the frequency of the occurrence of "flip-flop-flip" phenomenon is lower than that of the "flip-flop" phenomenon.

To synthesize the above results, for NCEP ensemble prediction products, the frequency difference of the "flip" phenomenon is not obvious in the ensemble average forecast and the corresponding set control forecast, however, the frequency of "flipflop" and "flip-flop-flip" phenomenon is obviously smaller than that of the corresponding set control forecast, which should be greatly concerned is that the difference between the two frequencies will become easier to distinguish when the forecast time is relatively long. The above phenomena show that the ensemble average forecast is quite low compared with the control forecast.

In this paper, the variation characteristics of "forecast jump" in different seasons are also analyzed, namely, the frequency size of "forecast jump" in the NCEP ensemble average forecast and control forecast in each season (Figure 6), and the difference of "jumping" frequency in ensemble average forecast and control forecast of NCEP
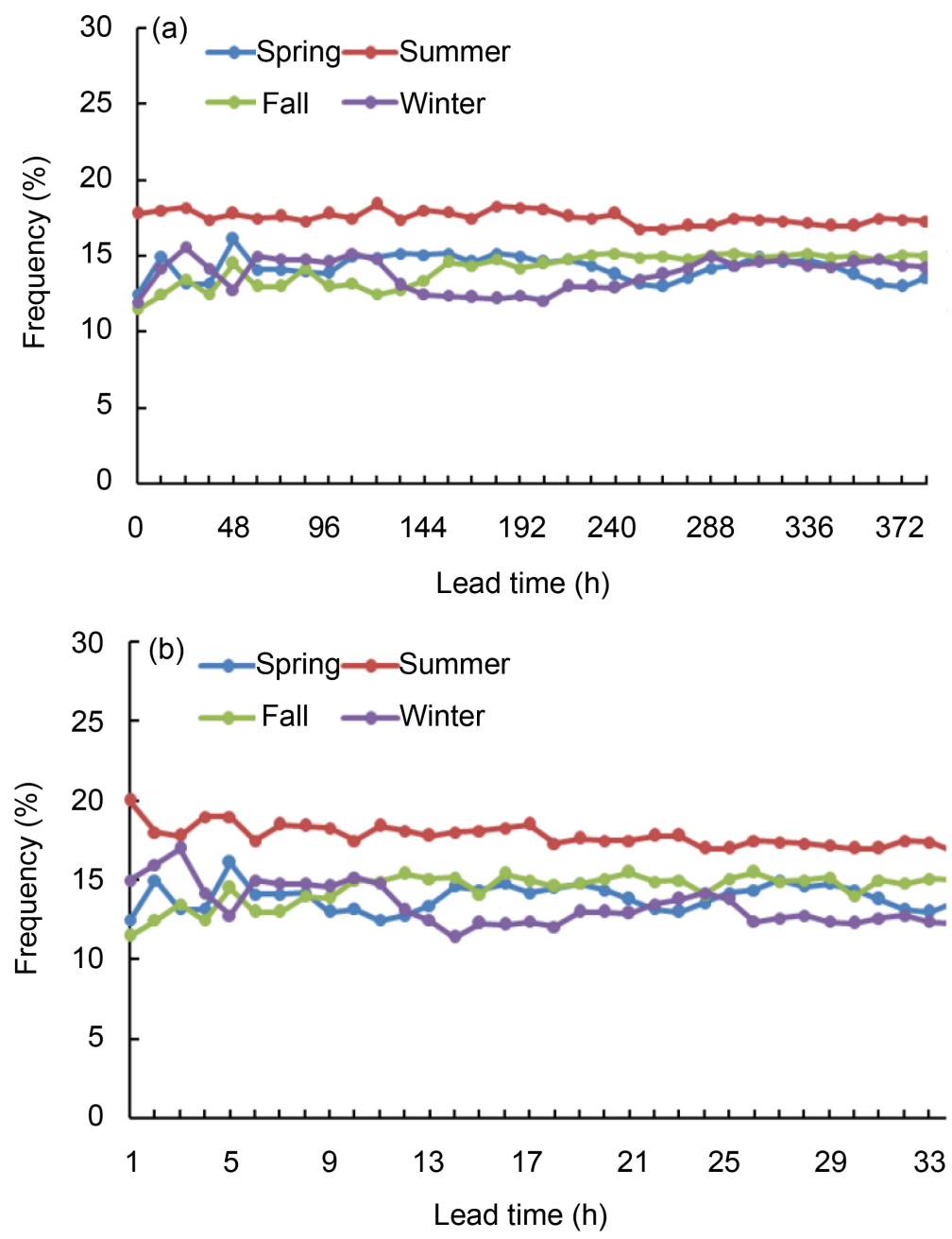

Figure 6. Seasonal variation characteristics of the frequency of "prediction jump" in the NCEP ensemble mean (a) and control forecast (b). 
ensemble in summer is compared. As shown in Figure 6(a) \& Figure 6(b), both ensemble average and its corresponding control forecast are the highest in the summer, and the lowest frequency of occurrence of "forecast jump" is winter, in spring and autumn, the frequency of "forecast jump" is located between the summer and winter, and the difference between the frequency of the occurrence of "forecast jump" in the spring and autumn period is small. In summer, with the extension of the forecast period, both the NCEP ensemble average forecast and the corresponding control forecast, the occurrence frequency of the "predicted jump" is shown to be a slight downward trend, at the same time, the change of the frequency of the ensemble average forecast and control forecast is roughly the same, and their frequency is between $15 \%-20 \%$; there is a slight difference between the frequency of the ensemble average forecast and the control forecast in the short range of prediction. In addition to the summer season, the other three seasons, that is, autumn, winter and spring, their "predicted jump" frequencies show a slight upward trend. But throughout the year, whether the NCEP ensemble average forecast and its corresponding control forecast, the frequency fluctuation of the phenomenon of "forecasting jump" is small, the above two kinds of forecast in the four seasons of the year, the frequency of "forecast jump" phenomenon is basically in between $10 \%-20 \%$. To sum up, the phenomenon of "prediction jump" has only limited seasonal sensitivity.

\section{Conclusions}

The verification method of NCEP ensemble prediction is introduced firstly in this paper, and then the concept and the statistical analysis method of the forecast jump are described. Finally, the advantages and disadvantages of the forecast consistency of the NCEP ensemble mean forecast and the corresponding control forecast, and the consistency and variability of "forecast jumps" in the ensemble mean forecast and control forecast are studied. The results are summarized as follows:

Two main attributes of NCEP ensemble forecast product inspection system are reliability and resolution. The main methods are: SPRD, RMSE, histogram, CRPS score, RPS score, BS score, etc.

Through the statistical analysis of average prediction time jump index, we found that, on average, in the NCEP ensemble forecast product, the time average prediction jump index increases with the increase of the forecast aging, and the actual forecast experience can reflect this phenomenon. At the same time, the consistency of ensemble average forecast is better than the corresponding control forecast.

The frequency of "flip", "flip-flop" and "flip-flop-flip", three different grades of "forecast jumping" phenomenon in the NCEP ensemble mean forecasts and corresponding control forecast decrease in turn. The frequency of occurrence of "forecast jump" phenomenon in the ensemble average and the corresponding control forecast at the same time is lower than the frequency of occurrence of "forecast jump" phenomenon in the ensemble average or the corresponding control forecast alone. For NCEP ensemble prediction products, generally speaking, the difference between the frequency of occurrence of "flip" phenomenon in the ensemble average forecast and its corresponding control forecast is small, however, especially in the longer forecast period, the frequency 
of "flip-flop" and "flip-flop-flip" phenomenon is obviously smaller than that of the corresponding control forecast. This fully shows that the inconsistent level average level of the ensemble average forecast is lower than the ensemble control forecast.

The frequency of occurrence of "forecast jump" phenomenon in the NCEP ensemble average forecast and its corresponding control forecast in summer is greater than in spring and autumn season, which is more than that in winter. But the occurrence frequency of "forecast jump" is limited to the sensitivity of the season. In summer, the frequency of "forecast jump" phenomenon is fluctuating by $17.5 \%$, which does not appear significant growth.

\section{Acknowledgements}

This work was supported by the National Meteorological Center's Youth Fund (No. Q201603), China.

\section{References}

[1] Persson, A. and Strauss, B. (1995) On the Skill and Consistency in Medium Range Weather Forecast Systems. ECMWF Newsletter, 70, 12-15.

[2] Zsoter, E., Buizza, R. and Richardson, D. (2009) "Jumpiness" of the ECMWF and Met Office EPS Control and Ensemble-Mean Forecasts. Monthly Weather Review, 137, 3823-3836. https://doi.org/10.1175/2009MWR2960.1

[3] Pappenberger, F., Cloke, H.L., Persson, A. and Demeritt, D. (2011) HESS Opinions “on Forecast (In)Consistency in a Hydro-Meteorological Chain: Curse or Blessing?” Hydrology and Earth System Sciences, 15, 2391-2400. https://doi.org/10.5194/hess-15-2391-2011

[4] Pappenberger, F., Bogner, K., Wetterhall, F., et al. (2011) Forecast Convergence Score: A Forecaster's Approach to Analyzing Hydro-Meteorological Forecast Systems. Advances in Geosciences, 29, 27-32. https://doi.org/10.5194/adgeo-29-27-2011

[5] Brooks, E.H. and Doswell III, A.C. (1993) New Technology and Numerical Weather Prediction-A Wasted Opportunity? Weather, 48, 173-177. https://doi.org/10.1002/j.1477-8696.1993.tb05877.x

[6] Kalnay, E. (2003) Atmospheric Modeling, Data Assimilation and Predictability. Cambridge University Press.

[7] Chen, J. and Xue, J. (2008) Heavy Rainfall Ensemble Prediction: Initial Condition Perturbation vs Multi-Physics Perturbation. Acta Meteorological Sinica, 23, 53-67.

[8] Buizza, R. (2008) The Value of Probabilistic Prediction. Atmospheric Science Letters, 9, 3642. https://doi.org/10.1002/asl.170

[9] Lashley, S.L., Fisher, L., Simpson, B.J., et al. (2008) Observing Verification Trends and Applying a Methodology to Probabilistic Precipitation Forecasts at a National Weather Service Forecast Office. 19th Conference on Probability and Statistics, New Orleans, Preprints.

[10] Persson, A. (2011) User Guide to ECMWF Forecast Products. ECMWF, Reading, 1-127.

[11] Park, Y., Buizza, P. and Leutbecher, M. (2008) TIGGE: Preliminary Results on Comparing and Combining Ensembles. Quarterly Journal of the Royal Meteorological Society, 134, 2029-2050. https://doi.org/10.1002/qj.334

[12] Ehret, U. (2010) Convergence Index: A New Performance Measure for the Temporal Stability of Operational Rainfall Forecasts. Meteorologische Zeitschrift, 19, 441-451.

https://doi.org/10.1127/0941-2948/2010/0480 
Submit or recommend next manuscript to SCIRP and we will provide best service for you:

Accepting pre-submission inquiries through Email, Facebook, LinkedIn, Twitter, etc. A wide selection of journals (inclusive of 9 subjects, more than 200 journals) Providing 24-hour high-quality service

User-friendly online submission system

Fair and swift peer-review system

Efficient typesetting and proofreading procedure

Display of the result of downloads and visits, as well as the number of cited articles Maximum dissemination of your research work

Submit your manuscript at: http://papersubmission.scirp.org/

Or contact acs@scirp.org 